\title{
Acute or chronic stress induce cell compartment-specific phosphorylation of glucocorticoid receptor and alter its transcriptional activity in Wistar rat brain
}

\author{
Miroslav Adzic, Jelena Djordjevic, Ana Djordjevic, Ana Niciforovic, Constantinos Demonacos ${ }^{1}$, \\ Marija Radojcic and Marija Krstic-Demonacos ${ }^{2}$ \\ Laboratory of Molecular Biology and Endocrinology, VINCA Institute of Nuclear Sciences, PO Box-522-MBE090, 11001 Belgrade, Serbia \\ ${ }^{1}$ School of Pharmacy and ${ }^{2}$ Faculty of Life Sciences, University of Manchester, Michael Smith Building, Oxford Road, Manchester, M13 9PT England, UK \\ (Correspondence should be addressed to M Krstic-Demonacos; Email: m.k.demonacos@manchester.ac.uk)
}

\begin{abstract}
Chronic stress and impaired glucocorticoid receptor (GR) feedback are important factors for the compromised hypothalamic-pituitary-adrenal (HPA) axis activity. We investigated the effects of chronic 21 day isolation of Wistar rats on the extrinsic negative feedback part of HPA axis: hippocampus (HIPPO) and prefrontal cortex (PFC). In addition to serum corticosterone (CORT), we followed GR subcellular localization, GR phosphorylation at serine 232 and serine 246, expression of GR regulated genes: GR, CRF and brain-derived neurotropic factor (BDNF), and activity of c-Jun N-terminal kinase (JNK) and Cdk5 kinases that phosphorylate GR. These parameters were also determined in animals subjected to acute $30 \mathrm{~min}$ immobilization, which was taken as 'normal' adaptive response to stress. In isolated animals, we found decreased CORT, whereas in animals exposed to acute immobilization, CORT was
\end{abstract}

markedly increased. Even though the GR was predominantly localized in the nucleus of HIPPO and PFC in acute, but not in chronic stress, the expression of GR, CRF, and BDNF genes was similarly regulated under both acute and chronic stresses. Thus, the transcriptional activity of GR under chronic isolation did not seem to be exclusively dependent on high serum CORT levels nor on the subcellular location of the GR protein. Rather, it resulted from the increased Cdk5 activation and phosphorylation of the nuclear GR at serine 232 and the decreased JNK activity reflected in decreased phosphorylation of the nuclear GR at serine 246. Our study suggests that this nuclear isoform of hippocampal and cortical GR may be related to hypocorticism i.e. HPA axis hypoactivity under chronic isolation stress.

Journal of Endocrinology (2009) 202, 87-97

\section{Introduction}

Response to neuroendocrine stress begins with the activation of the hypothalamic-pituitary-adrenal (HPA) axis leading to the increase in stress hormones glucocorticoids (GCs). These hormones mediate adaptation to stress and also regulate termination of the stress response through the intrinsic and extrinsic negative feedback action at the level of the HPA axis (De Kloet \& Reul 1987, Magarinos et al. 1987, Diorio et al. 1993). Feedback response in the extrinsic HPA axis structures hippocampus (HIPPO) and prefrontal cortex (PFC) is regulated by the GC receptor (GR), which, in the absence of hormone, resides in the cytoplasmic compartment as heterocomplex with heat shock proteins (Sanchez et al. 1990). Upon ligand binding, GR dissociates from the heterocomplex, translocates to the nucleus, and regulates neuronal target gene expression, including downregulation of the GR itself. However, under chronic stress this feedback sometimes becomes deregulated leading to the variety of maladaptive syndromes such as anxiety and various forms of depressive disorders (Sapolsky et al. 2000). In addition to regulating its own gene expression, GR also controls expression of other brain genes, such as corticotrophin-releasing hormone $(\mathrm{CRH})$, brain-derived neurotropic factor (BDNF), and cytokines (Goujon et al. 1997, Schulkin et al. 1998, Morsink et al. 2006, Schulte-Herbruggen et al. 2006). The GR transcriptional activity is dependent on the cell type, the sequence of the GC response elements, presence of coactivators or corepressors, and on post-translational modifications, such as phosphorylation (Weigel \& Moore 2007).

The rat GR is phosphorylated at threonine 171 (T171), serine 224 (S224), serine 232 (S232), or serine 246 (S246), which are all located in its N-terminal domain (Krstic et al. 1997). Several protein kinases phosphorylate GR at the specific sites, including c-Jun N-terminal kinase (JNK) and cyclin-dependent kinases (CDKs; Krstic et al. 1997, Ismaili \& Garabedian 2004, Kino et al. 2007). It has been reported that in heterologous systems, such as yeast strains that carry 
defective CDK homologues, GR exhibits decreased transcriptional activity suggesting that these kinases exert a stimulatory effect on GR. GR is also targeted by glycogen synthase kinase-3 that phosphorylates GR at Thr171 and this phosphorylation is shown to inhibit GR-mediated transcription, whereas mitogen-activated protein kinase (MAPK) family member JNK was reported to phosphorylate GR at S246 and also has a suppressive effect on GR function (Rogatsky et al. 1998, Ismaili \& Garabedian 2004). The GR phosphorylation was further reported to be involved not only in the regulation of its transcriptional activity, but also in the regulation of its subcellular localization and protein stability (Krstic et al. 1997, Webster et al. 1997, Rogatsky et al. 1998, Ismaili \& Garabedian 2004, Davies et al. 2008).

The role of the mentioned kinases, particularly of MAPKs, in the brain was related to the regulation of neurotransmission, synaptic plasticity, as well as to the consolidation of memory (Ortiz et al. 1995, Flood et al. 1998, Grewal et al. 1999, Sweatt 2001). For example, JNK pathway was reported to play a significant role in various brain regions as signalling mediator of different types of physical and psychological stressors (Meller et al. 2003, Shen et al. 2004). The capability of JNKs to phosphorylate GR implied their regulatory role in the central nervous system (CNS) stress response. In addition to that, recent reports document the role of one of the members of $\mathrm{Cdk}$ family, $\mathrm{Cdk} 5$, in the regulation of nervous system functions via GR-dependent mechanism (Kino et al. 2007, Cruz \& Tsai 2004).

In accordance with the above-mentioned reports in the current study, we exploited chronic 21 day isolation stress in Wistar male rats as a model of possible maladaptive stress. In this model, we analyzed GR subcellular localization, GR phosphorylation at serine 232 (GRS232) and at serine 246 (GRS246), and alterations in GR, CRF, and BDNF mRNA in the upper CNS regulatory structures HIPPO and PFC, which are related to the GR-mediated negative feedback regulation of the HPA axis activity. In parallel, the changes in protein expression of Cdk5 and its activators p35 and p25, as well as protein expression and phosphorylation of JNK1 and JNK2/3, were recorded. These parameters were also determined in Wistar males subjected to acute $30 \mathrm{~min}$ immobilization taken as 'normal' adaptive response to stress. Finally, the alterations in normal stress response after chronic stress experience were investigated by the combination of the two treatments mentioned above ('combined stress'). The combined stress model was used to test whether chronic stress caused irreversible alterations, which could potentially indicate that this form of stress is maladaptive.

\section{Materials and Methods}

\section{Animal care and treatment}

All experiments were performed in adult (3 months old) Wistar male rats (body mass 330-400 g) housed four per standard size cage with access to food (commercial rat pellets) and water ad libitum. Light was kept on, between 0700 and $1900 \mathrm{~h}$, and room temperature (RT) was kept at $20 \pm 2{ }^{\circ} \mathrm{C}$. All animal procedures were approved by the Ethical Committee for the Use of Laboratory Animals of the VINCA Institute of Nuclear Sciences, according to the guidelines of the EU registered Serbian Laboratory Animal Science Association (SLASA). For the analysis of the effects of stress, animals were divided into four groups: group I consisted of unstressed animals (control group); group II animals were exposed to acute immobilization for $30 \mathrm{~min}$; group III animals were subjected to chronic isolation stress, by housing them individually for 21 days; group IV was exposed to chronic isolation for 21 days followed by $30 \mathrm{~min}$ immobilization. All animals were killed immediately after the termination of the stress procedure.

\section{Corticosterone assay}

Blood from each animal was collected at the time of killing. Serum corticosterone (CORT) level was determined by using the OCTEIA CORT EIA kit according to the manufacturer's instructions (Immunodiagnostic Systems Ltd, Bolton, UK). Absorbance at $450 \mathrm{~nm}$ (reference $650 \mathrm{~nm}$ ) was determined by microplate reader (Wallac, VICTOR ${ }^{2} 1420$, PerkinElmer). CORT concentration $(\mathrm{ng} / \mathrm{ml})$ was determined using the standard curve.

\section{Preparation of cytoplasmic and nuclear extracts}

Frozen tissues were weighed and homogenized (1:2= tissue mass:vol) in ice-cold $20 \mathrm{mM}$ Tris- $\mathrm{HCl}(\mathrm{pH} \mathrm{7 \cdot 2)}$ buffer containing $10 \%$ glycerol, $50 \mathrm{mM} \mathrm{NaCl}, 1 \mathrm{mM}$ EDTA, $1 \mathrm{mM}$ EGTA, $2 \mathrm{mM}$ dithiothreitol, and protease inhibitors (20 $\mathrm{mM} \mathrm{Na}_{2} \mathrm{MoO}_{4}, 0.15 \mathrm{mM}$ spermin, $0.15 \mathrm{mM}$ spermidin, $0.1 \mathrm{mM}$ phenylmethylsulfonyl fluoride, $5 \mu \mathrm{g} / \mathrm{ml}$ antipain, $5 \mu \mathrm{g} / \mathrm{ml}$ leupeptin, $5 \mu \mathrm{g} / \mathrm{ml}$ aprotinin, $10 \mu \mathrm{g} / \mathrm{ml}$ trypsin inhibitor, and $3 \mathrm{mM}$ benzamidine) and phosphatase inhibitors (20 mM $\beta$-glycerophosphate, $5 \mathrm{mM} \mathrm{Na} \mathrm{P}_{2} \mathrm{O}_{7} \times 10 \mathrm{H}_{2} \mathrm{O}$, $2 \mathrm{mM} \mathrm{Na}_{3} \mathrm{VO}_{4}$, and $25 \mathrm{mM} \mathrm{NaF}$ ) with 20 strokes of Potter-Elvehjem teflon-glass homogenizer. Samples were centrifuged $10 \mathrm{~min}$ at $2000 \mathrm{~g}$ at $4{ }^{\circ} \mathrm{C}$, supernatants centrifuged for $1 \mathrm{~h}$ at $105000 \mathrm{~g}$, and final supernatants were used as cytoplasmic fraction. Pellets were washed in $0.5 \mathrm{ml}$ homogenization buffer, and centrifuged for $10 \mathrm{~min}$ at $2000 \mathrm{~g}$ at $4{ }^{\circ} \mathrm{C}$. Final pellets were weighed, resuspended $(1: 1$ = mass:vol) in the same buffer supplied with $0.5 \mathrm{M} \mathrm{KCl}$, incubated for $1 \mathrm{~h}$ in ice bath (with frequent vortexing), and centrifuged for $10 \mathrm{~min}$ at $8000 \mathrm{~g}$ at $4{ }^{\circ} \mathrm{C}$. Supernatant was used as nuclear extract (Spencer et al. 2000).

\section{Western-blot detection of the GR, Cdk5, and JNK proteins}

Protein concentration in the cytoplasm and nuclear fraction was determined by the method of Lowry (Lowry et al. 1951). Samples were mixed with denaturing buffer, according to 
Table 1 Specific primer sequences used for detection of glucocorticoid receptor (GR), corticotrophin-releasing hormone (CRH), and brainderived neurotropic factor (BDNF) gene expressions

\begin{tabular}{|c|c|c|c|c|}
\hline & Primer sequence & $\begin{array}{l}\text { Annealing } \\
\text { temperature }\left({ }^{\circ} \mathrm{C}\right)\end{array}$ & $\begin{array}{l}\text { Product } \\
\text { size }(b p)\end{array}$ & Reference \\
\hline \multicolumn{5}{|l|}{ cDNA product } \\
\hline GR & $\begin{array}{l}\text { 5'-TGCAAACCTCAATAGGTCGACCAG-3' } \\
\text { 5'-TAAACTGGGCCCAGTTTCTCTTGC-3' }\end{array}$ & 59 & 522 & Marlier et al. (1996) \\
\hline $\mathrm{CRH}$ & $\begin{array}{l}5^{\prime} \text {-TGATCCGCATGGGTGAAGAATACTTCCTC-3' } \\
5^{\prime} \text {-CCCGATAATCTCCATCAGTTTCCTGTTGCTG-3' }\end{array}$ & 70 & 394 & Baigent \& Lowry (2000) \\
\hline BDNF & $\begin{array}{l}5^{\prime} \text {-ATGGGACTCTGGAGAGCGTGAA-3' } \\
5^{\prime} \text {-CGC CAGCCA ATTCTC TTT TTGC-3' }\end{array}$ & 60 & 574 & Hatami et al. (2007) \\
\hline$\beta$-actin & $\begin{array}{l}\text { 5'-TTGTAACCAACTGGGACGATATGG-3' } \\
\text { 5'-GATCTTGATCTTCATGGTGCTAGG-3' }\end{array}$ & 57 & 764 & Zeng et al. (1999) \\
\hline
\end{tabular}

Laemmli (1970), boiled for $5 \mathrm{~min}$ at $100{ }^{\circ} \mathrm{C}$, and $60 \mu \mathrm{g}$ protein were subjected to electrophoresis on $7 \cdot 5 \%$ SDSPAGE. Subsequently, proteins were transferred onto PVDF membrane (Immobilon-P membrane, Millipore, Watford, UK) using a blot system (Transblot, Bio-Rad). The membranes were incubated in appropriate primary and secondary antibodies and signal was developed using enhanced chemiluminescence reagent (Pierce) and exposed to X-ray film (Fuji Photofilm, Bedfordshire, UK). GR M-20 antibody (Santa Cruz Biotechnology, Santa Cruz, CA, USA) was used to detect total GR (tGR) and phospho-GR antibody (Ser211) (Cell Signaling, New England Biolabs, Hertfordshire, UK) was used to detect GR phosphorylated at S211. The antiS246-P antibody was raised against the phosphorylated rat GR peptide LLIDENLLpSPLAGEDDP (amino acid residues 238-254) and custom made by Sigma (Davies et al. 2008). Anti-human Cdk5 and p35 polyclonal antibodies (Santa Cruz Biotechnology) were used to detect $\mathrm{Cdk} 5$ and its activator respectively. Anti-human JNK1/JNK2 monoclonal antibody (BD, Biosciences, Oxford, UK) and anti-phospho-SAPK/ JNK (Thr183/Tyr185) polyclonal antibody (Cell Signaling) were used to detect total or phosphorylated JNK respectively. Rabbit polyclonal anti- $\beta$-actin (Abcam, Cambridge, UK) was used to detect $\beta$-actin as a loading control. Blots were developed with goat anti-mouse or goat anti-rabbit HRPconjugated secondary antibody (Pierce). Anti- $\alpha$-tubulin (Sigma) and anti-NBS1 (GeneTex, Irvine, CA, USA) antibodies were used to analyze the purity of cytoplasmic and nuclear fractions respectively. Densitometry of protein bands on X-ray film was performed by Image J analysis PC software. Amounts of relative phosphorylated isoforms of all analyzed proteins were normalized to their total expression level and to $\beta$-actin.

\section{RT-PCR}

Total RNA was extracted using TRIzol Reagent (Invitrogen) according to the manufacturer's instructions. Briefly, tissues were weighed and homogenized in $1 \mathrm{ml}$ TRIzol Reagent per $100 \mathrm{mg}$ of tissue using Potter-Elvehjem teflon-glass homogenizer. Homogenates were then incubated at $30^{\circ} \mathrm{C}$ for
5 min in order to completely dissociate nucleoprotein complexes. Subsequently, $0 \cdot 2 \mathrm{ml}$ of chloroform was added and the homogenate was shaken vigorously for $15 \mathrm{~s}$ and incubated for $3 \mathrm{~min}$ at $30^{\circ} \mathrm{C}$. Samples were centrifuged at $12000 \mathrm{~g}$ for $15 \mathrm{~min}$ at $4{ }^{\circ} \mathrm{C}$. The aqueous phase, containing RNA, was mixed with $0.5 \mathrm{ml}$ of isopropanol, incubated at $30{ }^{\circ} \mathrm{C}$ for $10 \mathrm{~min}$, and centrifuged at $12000 \mathrm{~g}$ for $10 \mathrm{~min}$ at $4{ }^{\circ} \mathrm{C}$. Resulting RNA pellet was resuspended in $75 \%$ ethanol, centrifuged $\left(7500 \mathrm{~g}, 5 \mathrm{~min}, 4^{\circ} \mathrm{C}\right)$, air dried and dissolved in $100 \mu \mathrm{l} 0 \cdot 1 \%$ DEPC water, and stored at $-80^{\circ} \mathrm{C}$ until analysis. The concentration of RNA was determined by measuring the absorbance at $260 \mathrm{~nm}$ (A260) in a spectrophotometer. The ratio between the absorbance values at 260 and $280 \mathrm{~nm}$ was used to estimate RNA purity and all samples had A260/A280 ratio from 1.9 to 2 . The integrity of the purified RNA was determined by visualization of the $28 \mathrm{~S}$ and $18 \mathrm{~S}$ rRNA bands after electrophoresis of $2 \mu \mathrm{g}$ RNA of each sample on the $1 \cdot 5 \%$ agarose gel.

For the synthesis of cDNAs, a High-Capacity cDNA Reverse Transcription Kit (Applied Biosystems) was used according to the manufacturer's instructions. Namely, $2 \mu \mathrm{g}$ of total RNA was reverse transcribed using MultiScribe Reverse Transcriptase $(50 \mathrm{U} / \mu \mathrm{l})$ in the presence of $2 \mu \mathrm{l}$

Table 2 Serum corticosterone concentrations in control and stressed animals

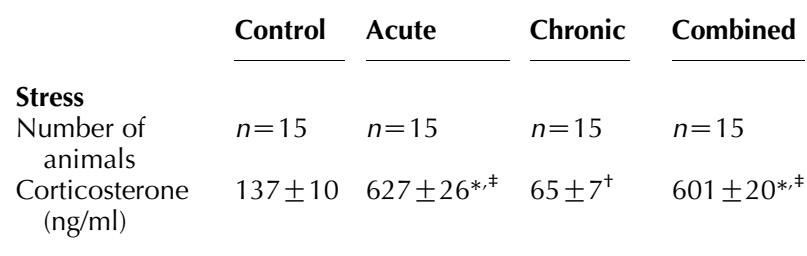

Serum corticosterone (CORT) is represented as mean \pm S.E.M. $(n=15)$. Statistical analysis by one-way ANOVA followed by Tukey's post hoc test indicated a significant increase in CORT concentration caused by acute immobilization stress $\left({ }^{*} P<0 \cdot 001\right.$ versus control) or combined stress (chronic isolation plus acute immobilization stress ${ }^{*} P<0.001$ versus control). The chronically isolated animals had significantly decreased CORT concentrations in respect to control level $\left({ }^{+} P<0 \cdot 01\right)$, acute $\left({ }^{\mp} P<0 \cdot 001\right)$ or combined stress animals $\left({ }^{\ddagger} P<0 \cdot 001\right)$. 
Random Primers, 0 $8 \mu \mathrm{l} 100 \mathrm{mM}$ dNTP Mix, $1 \mu \mathrm{l}$ RNase Inhibitor, and $10 \times \mathrm{RT}$ Buffer in the final volume of $20 \mu \mathrm{l}$. The cDNAs were stored at $-20{ }^{\circ} \mathrm{C}$.

For PCR, specific primers (Table 1) were designed to selectively amplify GR, CRH, BDNF, and $\beta$-actin as a housekeeping gene. For each set of primers, control experiments, involved varying the number of cycles, were performed to define the linear range for PCR amplification. As additional controls, PCRs lacking only template were run for each set of reactions. Appropriate dilutions of cDNA samples were mixed with PCR buffer containing $0 \cdot 2 \mathrm{mM}$ dNTPs, $2 \mathrm{mM} \mathrm{MgCl}_{2}, 0 \cdot 25 \mu \mathrm{M}$ primers for GR, CRF, BDNF, and $\beta$-actin, and $2 \mathrm{U}$ of Taq polymerase in a total volume of $25 \mu \mathrm{l}$. cDNAs were amplified in Eppendorf Mastercycler for 28 cycles using the following conditions: denaturation $94^{\circ} \mathrm{C} / 45 \mathrm{~s}$; annealing $59^{\circ} \mathrm{C} / 1 \mathrm{~min}$ (GR), $70^{\circ} \mathrm{C} / 1 \min (\mathrm{CRH}), 60^{\circ} \mathrm{C} / 1 \mathrm{~min}$ (BDNF), or $57^{\circ} \mathrm{C} / 1 \mathrm{~min}\left(\beta\right.$-actin); extension $72{ }^{\circ} \mathrm{C} / 1 \mathrm{~min}$; final extension $72{ }^{\circ} \mathrm{C} / 8 \mathrm{~min}$. PCR products were electrophoresed on $2 \%$ agarose gels together with a MassRuler Low Range DNA Ladder, 50-1500 bp (Fermentas, York, UK), and visualized under u.v. light using ethidium bromide. The intensity of PCR products was measured with an image analysis system GelDoc 1000 (Bio-Rad) and normalized using $\beta$-actin amplification as control.

\section{Statistical analysis}

Data are presented as mean \pm S.E.M. from three independent measurements of samples obtained from three separate groups of five animals (total number of animals 15 per experimental group). For establishing significant differences within one tissue under different stress, data were analyzed by the one-way ANOVA followed by the Tukey's post hoc test. Values were considered statistically significant if the $P$ value was $<0 \cdot 05$.

\section{Results}

Chronic stress lowers the level of CORT without impairing responsiveness to the subsequent acute stress

CORT concentration was detected using a commercial CORT kit (Table 2). In the control group of Wistar rats, the CORT level was $137 \mathrm{ng} / \mathrm{ml}$, and was in the same range as reported by other authors (Merino et al. 2000, Wren et al. 2002). As expected, the acute (30 min) exposure to the high-intensity physical-emotionalpsychosocial stress, such as immobilization, resulted in a significant increase in serum CORT levels to $627 \mathrm{ng} / \mathrm{ml}$. On the contrary, chronic isolation for 21 days (lowintensity but long-term psychosocial stress) led to significant decrease in CORT serum levels to $65 \mathrm{ng} / \mathrm{ml}$ in accordance with previous reports (Sanchez et al. 1998, Malkesman et al. 2006). When the chronically stressed animals were subsequently subjected to acute immobilization (i.e. combined stress), serum CORT increased to a similar level as that observed after acute stress (Table 2).

\section{Chronic stress does not alter GR subcellular localization in HIPPO}

Effects of acute and combined stresses (that resulted in high CORT levels - see Table 2) on hippocampal tGR level were reflected in the significant reduction in cytoplasmic and increase in nuclear tGR (Fig. 1D). By contrast, both cytoplasmic and nuclear HIPPO tGR protein levels were unchanged after chronic stress. Although we can detect the GR in both compartments, the results in Fig. 1 showed that acute and combined stresses induced nuclear translocation of the receptor, whereas chronic stress does not induce GR translocation (Fig. 1).
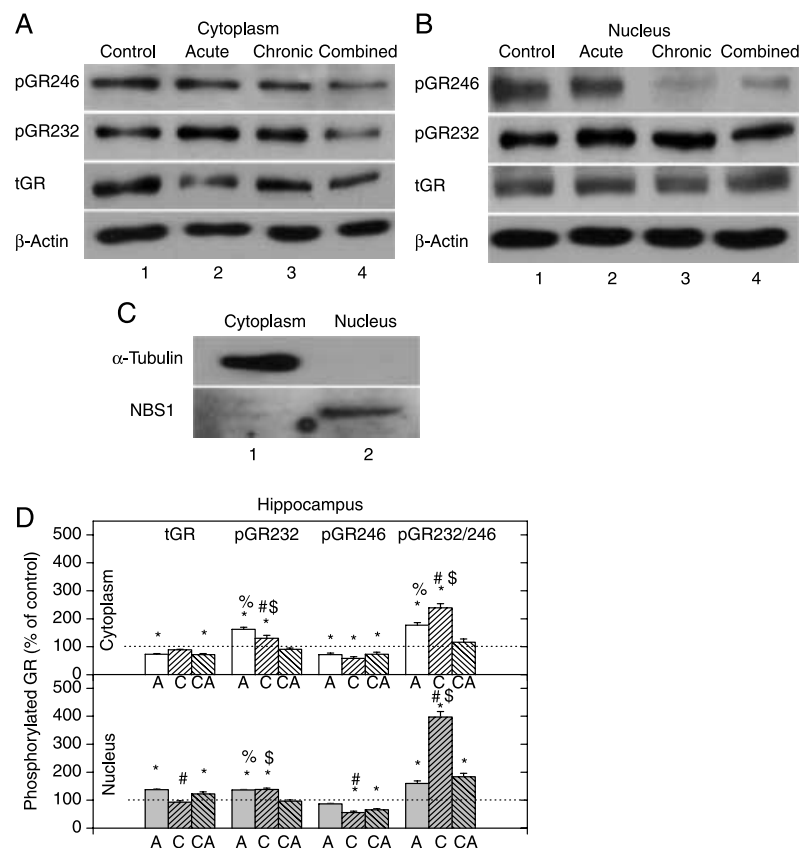

Figure 1 Western-blot $(W B)$ experiment demonstrating the effects of acute immobilization $(A)$, chronic isolation $(C)$, or combined stress (CA) on the levels of glucocorticoid receptor (GR) and its phosphoisoforms in the cytoplasm and nucleus of hippocampus: (A and B) GR phosphorylation at S246 (pGR246) or S232 (pGR232). Cell lysates were resolved by SDS-PAGE and probed with antibodies against total GR (tGR), pGR246, pGR232, or actin as a loading control. (C) The purity of subcellular fractions was assayed by anti- $\alpha$-tubulin or anti-NBS1 antibodies for the cytoplasmic or nuclear compartments respectively. (D) Immunoreactivities of the cytoplasmic and nuclear GR, pGR232, and pGR246 (normalized to actin) and ratio of pGR232/pGR246 (normalized to GR in each compartment) are expressed as mean \pm S.E.M. (as described under Materials and Methods); $n=15$ animals per experimental group. Asterisks indicate significant differences between treated groups. Statistical analysis was performed using one-way ANOVA followed by the Tukey's post hoc test $\left({ }^{*} P<0 \cdot 05\right.$, stress versus control; ${ }^{\#} P<0 \cdot 05$, acute versus chronic; ${ }^{\%} P<0 \cdot 05$, acute versus combined; ${ }^{\$} P<0.05$ chronic versus combined). 
A

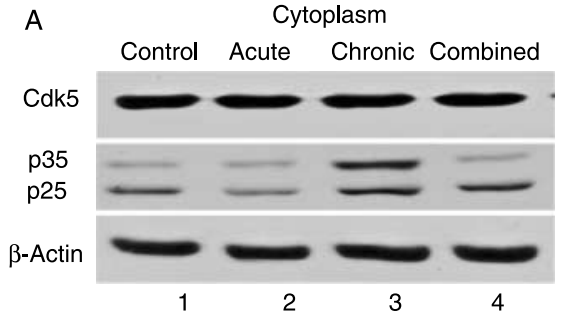

B

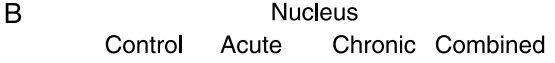

Cdk5

p35

p25

$\beta$-Actin
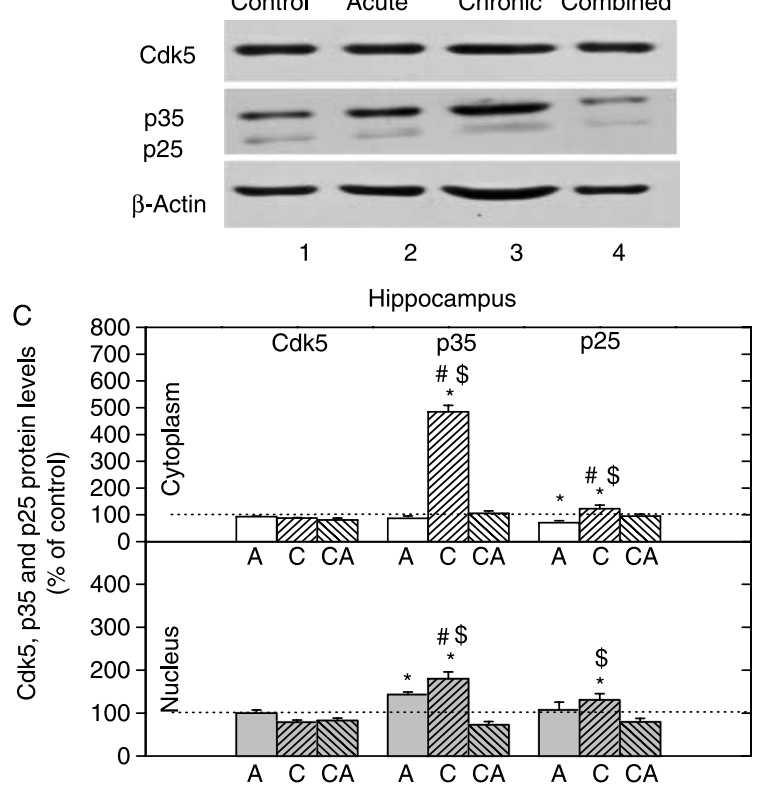

Figure 2 Western-blot (WB) experiment demonstrating the effects of acute immobilization $(A)$, chronic isolation $(C)$, or combined stress (CA) on the levels of Cdk5, its activators p35 and p25 in the cytoplasm and nucleus of hippocampus: (A and B) Cdk5, p35, and p25 proteins in the cytoplasm (A) and nucleus (B) of hippocampus. Hippocampal lysates were resolved by SDS-PAGE and membranes were probed with respective antibodies that recognized indicated proteins and with anti-actin antibody as a loading control. (C) Immunoreactivities of Cdk5, p35, and p25 proteins in the cytoplasm and nucleus of hippocampus are given as mean \pm s.E.M. (as described under Materials and Methods); $n=15$ animals per experimental group. Statistical analysis was performed using one-way ANOVA followed by the Tukey's post hoc test and labelled as in the Fig. 1.

\section{GR phosphorylation at S232 is dominant over the S246} phosphorylation independent of the type of stress or cellular compartment

In order to analyze effects of stress on the GR function in HIPPO, we analyzed its phosphorylation status. Cyclindependent protein kinases (CDKs) phosphorylate S224 and S232 of the rat GR, whereas JNK pathway has been shown to phosphorylate S246 amino acid. It has been suggested that phosphorylation of these residues is of importance in the regulation of the GR transcriptional activity and the target gene specificity (Blind \& Garabedian 2008, Davies et al. 2008). In the next series of experiments, we observed the increased level of GRS232 phosphorylation in both cytoplasmic and nuclear HIPPO extracts following acute and chronic stresses (Fig. 1A, B second panel, and D). In animals subjected to the combined stress, GRS232 was similar to the control level in both compartments (Fig. 1D). On the other hand, both cytoplasmic and nuclear GRS246 phosphorylation levels were lower than control in most samples (Fig. 1A, B first panel, and D). The pGR232/246 ratio was determined in order to quantitatively compare the extent of GR phosphorylation at S232 or S246 (Fig. 1D). The ratio values above $100 \%$ indicated the dominance of GRS232 phosphorylation, while values below 100\% suggested the prevalence of GRS246 phosphorylation. This approach indicated that GR phosphorylation at S232 is predominant compared with that at S246, independent of the type of stress or cellular compartment analyzed. The only exception was that of the cytoplasmic GR in the combined stress, which was equally phosphorylated at both S232 and S246 (Fig. 1D). The highest pGR232/246 ratio was found in the group of chronically stressed animals in both compartments.
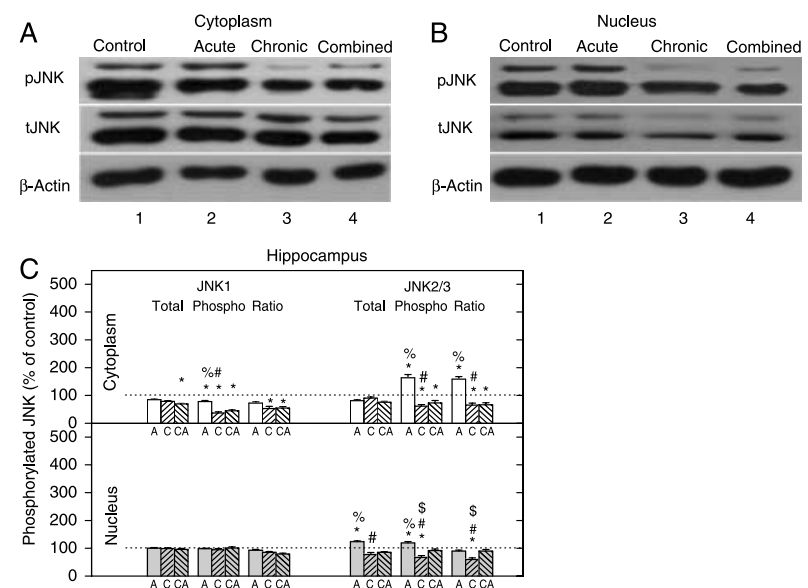

Figure 3 Western-blot $(W B)$ experiment demonstrating the effects of acute immobilization (A), chronic isolation (C), or combined stress (CA) on the levels of JNKs and their phosphoisoforms in the cytoplasm and nucleus of hippocampus: (A and B) JNK proteins (tJNK1 at $46 \mathrm{kDa}$ and tJNK2 and 3 at $54 \mathrm{kDa}$ ) and their phosphoisoforms (pJNK1, pJNK2 and 3) in the cytoplasm (A) and nucleus (B) of hippocampus. Hippocampal lysates were resolved by SDS-PAGE and membranes were probed with antibody that recognized all three tJNKs, or the specific JNK phosphoisoforms (pJNK1, 2, and 3) and with anti-actin antibody as a loading control. (C) Immunoreactivities of tJNK1, tJNK2/3, pJNK1, pJNK2/3, and their ratios (pJNK1/tJNK1 and pJNK2/3/tjNK2/3) in the cytoplasm and nucleus of hippocampus are given as mean \pm S.E.M. (as described under Materials and Methods); $n=15$ animals per experimental group. Statistical analysis was performed using one-way ANOVA followed by the Tukey's post hoc test and labelled as in Fig. 1. 

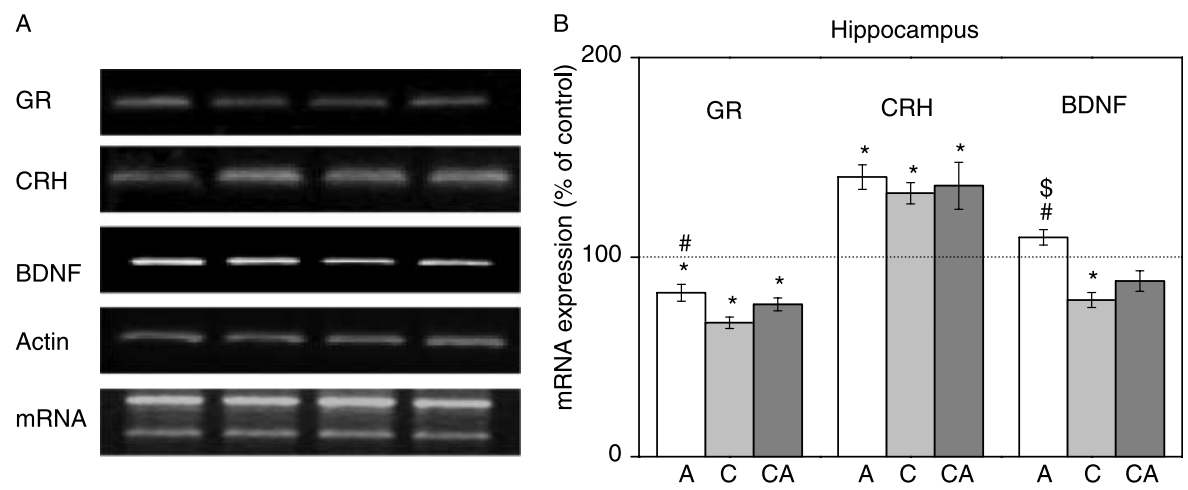

Figure 4 Effects of acute, chronic, and combined stresses on GR, CRH, and BDNF gene expressions in the rat hippocampus measured by RT-PCR (A). A semi-quantitative evaluation of gene expressions from six independent experiments is shown in (B). Data are presented as mean \pm s.E.M., $n=15$ animals per experimental group. Statistical analysis was performed using one-way ANOVA followed by the Tukey's post hoc test and labelled as in Fig. 1.

Changes in expression of the Cdk5, p35, and 25 proteins in HIPPO

The expression level of Cdk5 was not changed by any of the three stresses in either of the cell compartments (Fig. 2). The level of p35 protein, an activator of Cdk5, was markedly elevated by chronic stress in the cytoplasm, while its nuclear level was increased by acute and chronic stresses. In addition to that, p25 protein, which is a more active form of $\mathrm{Cdk} 5$ activator, was somewhat increased only under chronic stress in both cell compartments (Fig. 2).

JNK2/3 activity is upregulated by the acute and downregulated by the chronic and combined stresses, whereas JNK1 is inhibited under all stress conditions in HIPPO

In the next set of experiments, we analyzed the levels and activity of the JNK1 detected as $46 \mathrm{kDa}$ protein ( $\mathrm{t} J \mathrm{NK} 1)$ and JNK 2 and 3 detected as $54 \mathrm{kDa}$ proteins (tJNK2 and 3, Fig. 3C). These experiments showed that the levels of all three JNK proteins in both cellular compartments were mainly unchanged (Fig. 3C). The level of pJNK1 isoform in the cytoplasm of HIPPO was significantly reduced by all three stresses in comparison with control (Fig. 3A and C), while the nuclear level of pJNK1 isoform in HIPPO remained unaltered by any of the stresses (Fig. 3B and C). The level of pJNK2/3 was significantly elevated in the cytoplasm of HIPPO by acute stress (Fig. 3A-C), while it was significantly reduced by either chronic or combined stress. The nuclear level of $\mathrm{pJNK} 2 / 3$ isoforms was reduced by the chronic stress (Fig. 3B and C). The ratio of pJNK1 to tJNK1 (pJNK1/tJNK1) indicated that cytoplasmic JNK1 activity was low in all types of stress (Fig. 3C). By contrast, the ratio of cytoplasmic pJNK2/3/tJNK2/3 was markedly high in acute stress, while it was low in two other types of stress and in nucleus this ratio was decreased under chronic stress (Fig. 3C).
Changes in the expression of the GR, CRH and BDNF genes in $\mathrm{HIPPO}$

The role of phosphorylated GR in the regulation of gene expression was tested on GR-regulated genes namely, the GR (Hermann \& Spencer 1998), the CRH (Lu \& Cidlowski 2006), and the BDNF (Tapia-Arancibia et al. 2004). The chosen genes are known to be involved in the regulation of several stress-related CNS activities. GR regulates HPA axis activity, while $\mathrm{CRH}$ and BDNF are involved in the modulation of learning, memory processing, and synaptic plasticity of HIPPO and PFC (Givalois et al. 2000, Marmigere et al. 2003). As shown in Fig. 4 (lane 1), expression of GR gene was significantly decreased by all three types of stress with a dominant change under chronic stress. Expression of BDNF gene was significantly inhibited under chronic stress, while combined and acute stresses did not affect its expression with respect to the control. On the contrary, the expression of $\mathrm{CRH}$ was significantly increased by all three types of stress.

Chronic stress influences GR subcellular localization in the PFC in the opposite manner than the acute and combined stresses

Low cytoplasmic and concomitantly high nuclear tGR level was observed in PFC in acute stress (i.e. high CORT) (Fig. 5A, B third panel, and C). Under chronic stress, tGR protein level was increased in both compartments, while in combined stress this increase was detected only in the nucleus (Fig. 5A-C). The statistical intergroup analysis indicated significant compartmental differences in the tGR protein level, between acute or combined stress on one side and chronic stress on the other (Fig. 5C).

\section{The PFC GR phosphorylation on S232 is dominant under} chronic stress

The cytoplasmic GRS232 phosphorylation level was increased in chronic and combined stresses (Fig. 5A). 
A

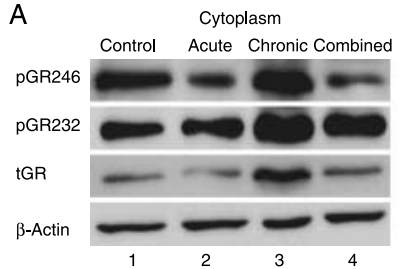

B

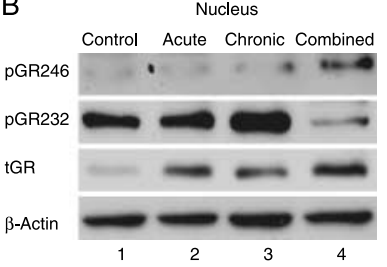

Prefrontal cortex

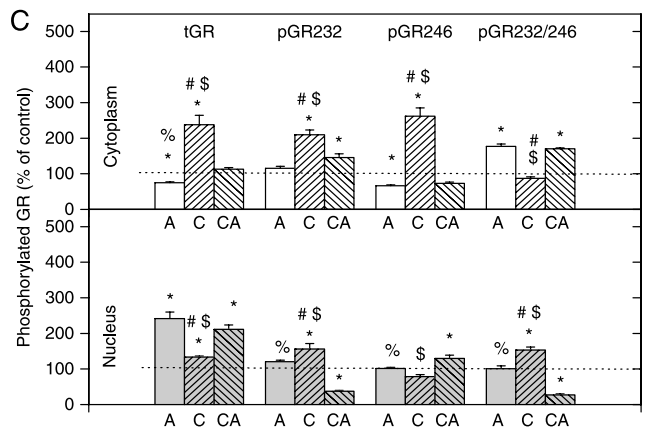

Figure 5 Western-blot (WB) experiment demonstrating the effects of acute immobilization (A), chronic isolation (C), or combined stress (CA) on the levels of glucocorticoid receptor (GR) and its phosphoisoforms in the cytoplasm and nucleus of prefrontal cortex: (A and B) GR phosphorylated at S246 (pGR246) or S232 (pGR232). Cell lysates were resolved by SDS-PAGE and probed with antibodies against total GR (tGR), pGR246, pGR232, or actin as a loading control. (C) Immunoreactivities of the cytoplasmic and nuclear GR, pGR232, and pGR246 (normalized to actin) and ratio of pGR232/pGR246 (normalized to GR in each compartment) are expressed as mean \pm S.E.M. (as described under Materials and Methods); $n=15$ animals per experimental group. Asterisks indicate significant differences between treated groups. Statistical analysis was performed using one-way ANOVA followed by the Tukey's post hoc test and labelled as in Fig. 1.

The nuclear GRS232 phosphorylation level was increased in chronically stressed animals and significantly decreased in combined stress (Fig. 5B). GR phosphorylation at S246 was markedly increased in cytoplasm under chronic stress and mostly unchanged by other treatments (Fig. 5A, B first panel, and C). pGR232/246 ratio was elevated in the cytoplasm in acute and combined stresses, while in the nucleus, this ratio was elevated under chronic stress and diminished in combined stress.

Changes in the expression of $C d k 5, p 35$, and $p 25$ proteins in PFC

The cytoplasmic levels of Cdk5 and p35 proteins were significantly increased only by chronic stress, whereas their nuclear levels were increased only by combined stress (Fig. 6). The cytoplasmic level of p25 was increased by both chronic and combined stresses, while its nuclear level was increased only by chronic stress (Fig. 6). Our results indicated that Cdk5 activity might be increased in both cell compartments under chronic and combined stresses.
The phosphorylation of JNK1 and JNK2/3 in the cytoplasmic and nuclear compartment of the PFC was reduced under chronic stress

The total JNK1 and JNK2/3 protein levels were mostly unaltered by any type of stress in both compartments (Fig. 7C). Both cytoplasmic and nuclear pJNK1 and pJNK2/3 levels were downregulated in chronic stress and unaltered under combined stress (Fig. 7C). Distinctly enhanced cytoplasmic pJNK2/3 protein level was observed only in the case of acute stress (Fig. 7A and C). The pJNK/ tJNK ratio indicated that the activity of both JNK1 and JNK2/3 was reduced under chronic stress in both compartments (Fig. 7C), while in acute stress increased cytoplasmic pJNK2/3/tJNK2/3 ratio was observed, suggesting that JNK2/3 but not JNK1 is activated in the acute stress.

Changes in the expression of GR, CRH, and BDNF genes in PFC

The expression of GR gene in PFC was downregulated under chronic stress. On the contrary, CRH and BDNF genes were
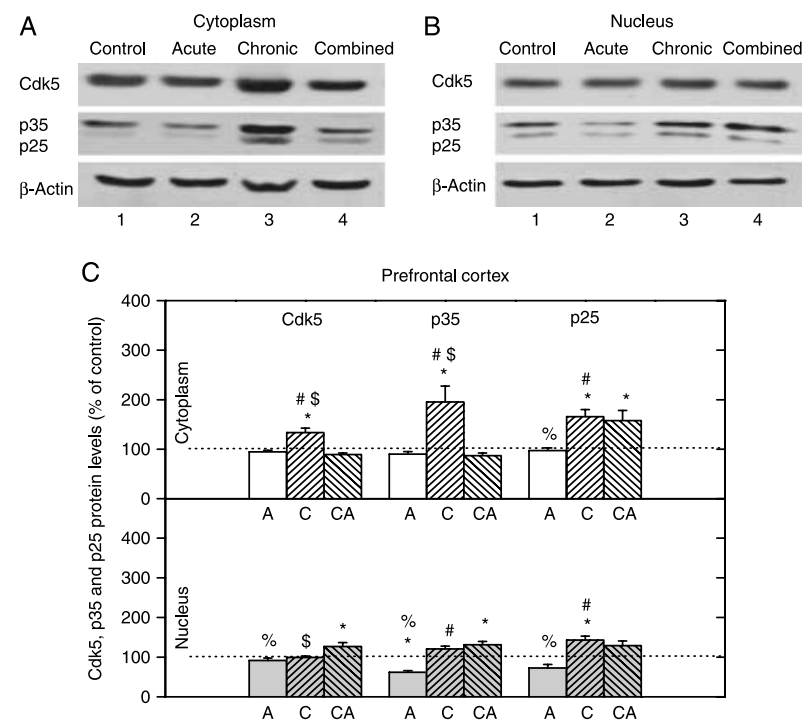

Figure 6 Western-blot $(W B)$ experiment demonstrating the effects of acute immobilization (A), chronic isolation $(C)$, or combined stress (CA) on the levels of Cdk5, its activators p35 and p25 in the cytoplasm and nucleus of prefrontal cortex: (A and B) Cdk5, p35, and p25 proteins in the cytoplasm $(A)$ and nucleus $(B)$ of prefrontal cortex. Prefrontal cortex lysates were resolved by SDS-PAGE and membranes were probed with respective antibodies that recognized these proteins and with anti-actin antibody as a loading control. (C) Immunoreactivities of Cdk5, p35, and p25 proteins in the cytoplasm and nucleus of prefrontal cortex are given as mean \pm s.E.M. (as described under Materials and Methods); $n=15$ animals per experimental group. Statistical analysis was performed using one-way ANOVA followed by the Tukey's post hoc test and labelled as in the Fig. 1. 

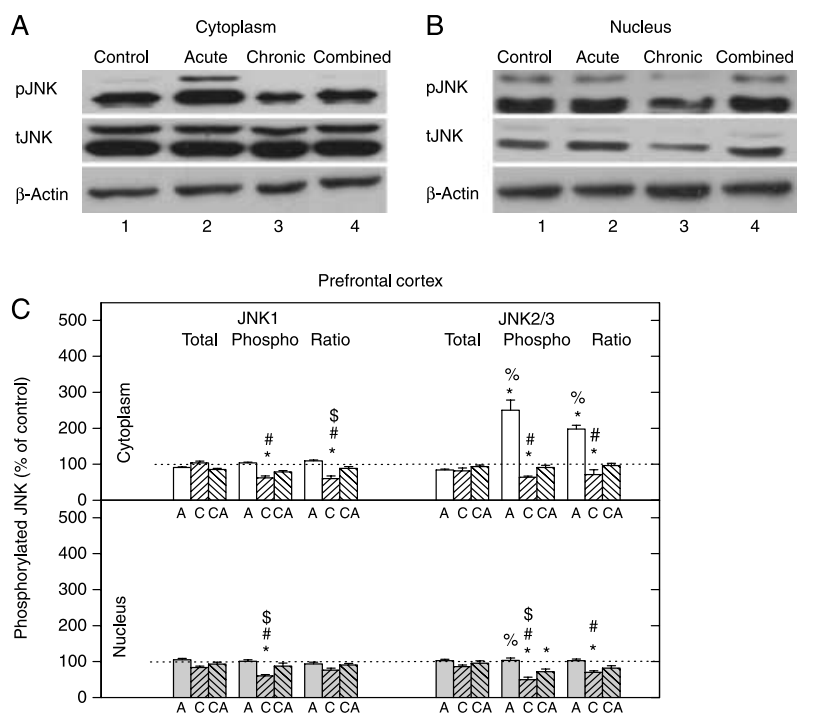

Figure 7 Western-blot (WB) experiment demonstrating the effects of acute immobilization, chronic isolation, or combined stress on the levels of JNKs and their phosphoisoforms in the cytoplasm and nucleus of prefrontal cortex (PFC). (A and B) JNK proteins (tJNK1 at $46 \mathrm{kDa}$ and tJNK2 and 3 at $54 \mathrm{kDa}$ ) and their phosphoisoforms (pJNK1, pJNK2, and 3) in cytoplasm (A) and nucleus (B) of prefrontal cortex. Cell lysates were resolved by SDS-PAGE and membranes were probed with antibody that recognized all three tJNKs, or the specific JNK phosphoisoforms (pJNK1, 2 and 3) and with anti-actin antibody as a loading control. (C) Immunoreactivities of tJNK1, tJNK2/3, pJNK1, pJNK2/3, and their ratios (pJNK1/tjNK1 and pJNK2/3/tJNK2/3) in the cytoplasm and nucleus of prefrontal cortex are given as mean \pm s.E.M. (as described under Materials and Methods); $n=15$ animals per experimental group. Statistical analysis was performed using one-way ANOVA followed by the Tukey's post hoc test and labelled as in the Fig. 1.

both upregulated under the same stress conditions (Fig. 8A and $\mathrm{B}$ lane 2 and 3 ). In response to acute and combined stresses, expression of all three genes was not significantly changed, with the exception of BDNF that was downregulated in the combined stress.
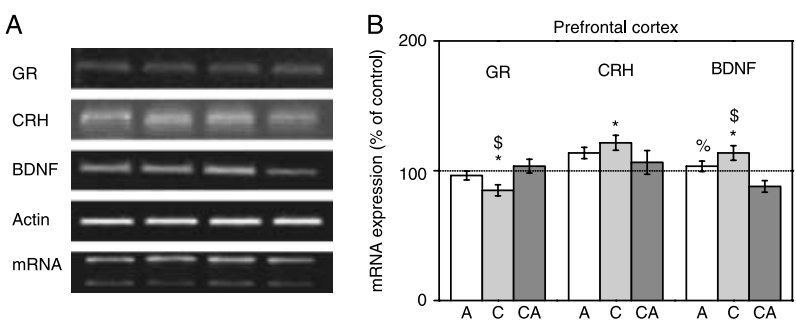

Figure 8 Effects of acute, chronic, and combined stresses on GR, $\mathrm{CRH}$, and BDNF gene expressions in the rat prefrontal cortex measured by semi-quantitative PCR (A). A semi-quantitative evaluation of gene expressions from six independent experiments is shown in (B). Data are presented as mean \pm S.E.M.; $n=15$ animals per experimental group. Statistical analysis was performed using one-way ANOVA followed by the Tukey's post hoc test and labelled as in the Fig. 1.

\section{Discussion}

It has been postulated that the maladaptive response of the CNS to neuroendocrine stress may emerge from its compromised termination, which is at least in part based on the impaired GR negative feedback in the upper parts of the CNS. Such alterations were observed under conditions of chronic stress (Chrousos \& Kino 2007). In the present in vivo study, we exploited the Wistar rat model to investigate chronic stress-dependent GR alterations in HIPPO and PFC, the two CNS structures known to participate in the HPA axis homeostatic regulation. We analyzed stress-dependent GR redistribution and its transcriptional activity after the chronic 21 day social isolation that represented potentially maladaptive stress. In animals subjected to this type of stress, we found a decreased level of CORT in the blood serum compared with the control animals. This finding is in accordance with the data of other authors showing HPA axis hypoactivity in Wistar rats under similar conditions (Sanchez et al. 1998, Malkesman et al. 2006). In the group of animals exposed to acute $30 \mathrm{~min}$ immobilization (Garcia et al. 2000), applied either solely or after the chronic stress, we found a significantly increased level of serum CORT. The prominent increase in CORT after acute immobilization was expected, since this type of stress is of high intensity (Garcia et al. 2000). Thus, animals with previous chronic stress experience did not exhibit compromised HPA axis responsiveness to the subsequent acute stress as concluded from the observed hormone levels.

The analysis of the tGR protein levels in HIPPO indicated that in accordance with the well-known 'dogma' of the molecular mechanism of GR action (Nishi \& Kawata 2006), this protein was translocated from the cytoplasmic to the nuclear compartment when CORT levels were elevated, i.e. under acute or combined stress. In agreement with that, under the chronic stress, when CORT levels were low, tGR levels in the cytoplasm and nucleus of HIPPO remain indistinguishable from the control. Thus, our results suggested that chronic stress did not compromise the ability of GR to undergo nuclear translocation upon subsequent acute stress, i.e. when hormone level is increased.

However, under the chronic stress, despite of unaltered level of the nuclear tGR, this protein exhibited significant transcriptional activity regarding the expression of its own gene, as well as the expression of CRF and BDNF genes, which are two other well-known GR targets (Tapia-Arancibia et al. 2004, Lu \& Cidlowski 2006). This nuclear tGR activity under chronic stress was comparable with the one observed under acute stress, i.e. when CORT levels were high and increased levels of the GR were found in the nucleus. Namely, under both acute and chronic stresses, similar downregulation of GR gene and upregulation of $\mathrm{CRH}$ gene were detected. Moreover, while BDNF was unaltered by acute stress, it was downregulated under chronic stress. These findings suggested that the transcriptional 
activity of GR did not seem to be regulated solely by the levels of hormone. In order to further investigate this process, we followed the GR phosphorylation status that is one important level of control of the GR transcriptional activity (Krstic et al. 1997, Rogatsky et al. 1998, Ismaili \& Garabedian 2004, Davies et al. 2008). Specifically, we monitored GR phosphorylation at the two GR amino acids S232 and S246, which are known to be modified by CDK and JNK kinases respectively (Orti et al. 1992, Krstic et al. 1997, Webster et al. 1997, Rogatsky et al. 1998, Ismaili \& Garabedian 2004, Davies et al. 2008). We hypothesized that the pattern of GR phosphorylation, particularly that of the nuclear GR, might be related to GR transcriptional activity at the three target genes studied.

Under chronic stress, we observed an increase in GR phosphorylation at S232 in both cytoplasmic and nuclear compartments of HIPPO, which corresponded well with the elevated levels of Cdk5 activator proteins p35 and p25. Considering that association of Cdk5 with p35 as well as its association with $\mathrm{p} 25$ is critical for kinase activation (Tsai et al. 1994), our results indicated that Cdk5 activity could be increased in the both cell compartments under chronic stress. Further analyses indicated that at the same time chronic stress decreased GR phosphorylation at S246 in both cell compartments. The decrease in nuclear GRS246 phosphoisoform level corresponded well with downregulation of JNK $2 / 3$ activities under the chronic stress. Taken together, these results suggest that increase in GRS232 phosphorylation may be at least in part due to diminished phosphorylation of GR at S246, i.e. higher activity of Cdk5 relative to that of JNKs at the level of GR. This is in accordance with data of Ismaili \& Garabedian (2004) that the decrease in GRS246 phosphorylation may stimulate that at GRS232. As an additional measure of the GR phosphorylation status, we determined GRS232/GRS246 ratio and observed the clear prevalence of nuclear GRS232 isoform under chronic stress. This GR phosphoisoform may be important for BDNF and GR gene repressions and $\mathrm{CRH}$ gene activation in chronic stress where hormone levels are low. Thus, increased phosphorylation of hippocampal GR at S232 and its decreased phosphorylation at S246 may be related to GR transcriptional activity, HPA axis hypoactivity, and compromised negative feedback regulation (Herman \& Spencer 1998). It is important to mention that the pattern of hippocampal GR phosphorylation under chronic stress was reversed by subsequent acute stress.

In contrast to unchanged levels of tGR in HIPPO under chronic stress, this stress caused increase in GR protein levels in both compartments of PFC and its accumulation in the cytoplasm. Such differences in response of PFC to chronic stress compared with those of HIPPO could be due to alternative signalling pathways operating in these CNS substructures (Mizoguchi et al. 2003) or altered level/activity of tGR-interacting proteins (chaperones, kinases, components of proteasome, and other cofactors). Nevertheless, under acute or combined stress, the compartmental distribution of tGR in PFC was similar to that of HIPPO, i.e. GR exhibited translocation to the nucleus upon CORT increase. Thus, likewise in HIPPO, GR translocation to the nucleus of PFC was not compromised by the previous chronic stress exposure.

Similarly to our findings in HIPPO of chronically stressed animals, the tGR in the nuclear compartment of PFC also exhibited transcriptional activity, since the expression of cortical GR gene was attenuated, whereas expression of CRF and BDNF genes was upregulated. The magnitude of these changes in gene expression in PFC was somewhat smaller compared with the one observed in HIPPO, which may be explained by the relatively lower initial level of tGR in PFC (Cerqueira et al. 2005). The analysis of GR phosphorylation status in PFC of chronically stressed animals revealed, again, the presence of increased levels of GRS232 form primarily in the nucleus. Its presence in the nucleus corresponded well with the elevated levels of Cdk5, as well as of its activator proteins p35 and p25. The nuclear GRS232 prevailed over nuclear S246 phosphoisoform, which again may be due to decreased activity of JNKs. In contrast to HIPPO, the pattern of cortical GR phosphorylation under chronic stress was not reversed by subsequent acute stress, indicating prevalence of S246 phosphorylation that correlated with BDNF gene repression.

In summary, our data indicated that the regulation of the expression of the GR, CRH, and BDNF genes in HIPPO and PFC under chronic stress did not seem to be exclusively dependent on the predominant nuclear positioning of GR protein determined by high serum CORT levels. This finding suggests that cell signaling through GR phosphorylation may be of special importance under low GC's level, i.e. low GR occupancy with GCs as recently also reported by Chen et al. (2008). Based on these observations, future efforts should be directed towards detailed analyses of transcriptional activity of individual GR phosphoisoforms. In particular, it will be important to provide more evidence regarding the role of S232 phosphoisoform of the GR in chronic stress-induced hypocorticism and HPA axis hypoactivity. In that view molecular signals emerging from cell membrane receptors or those generated internally e.g. from mitochondria should be considered as potential regulators of the GR activity. Understanding of those mechanisms would be of immense help for the selection of pharmaceutical agents aiming to restore chronic stress-disturbed homeostasis of HPA axis.

\section{Declaration of interest}

There is no conflict of interest.

\section{Funding}

This work was supported by the Wellcome Trust (Research Fellowship 069024) and the Ministry of Sciences of Serbia (Grant ON143042B). 


\section{Acknowledgements}

We gratefully acknowledge the Wellcome Trust (Research Fellowship 069024) and the Ministry of Sciences of Serbia (Grant ON143042B) for the financial support of the study.

\section{References}

Baigent SM \& Lowry PJ 2000 mRNA expression profiles for corticotrophinreleasing factor (CRF), urocortin, CRF receptors and CRF-binding protein in peripheral rat tissues. Journal of Molecular Endocrinology 25 43-52.

Blind RD \& Garabedian MJ 2008 Differential recruitment of glucocorticoid receptor phospho-isoforms to glucocorticoid-indued genes. Journal of Steroid Biochemistry Molecular Biology 109 150-157.

Cerqueira JJ, Pêgo JM, Taipa R, Bessa JM, Almeida OF \& Sousa N 2005 Morphological correlates of corticosteroid-induced changes in prefrontal cortex-dependent behaviors. Journal of Neuroscience 25 7792-7800.

Chen W, Dang T, Blind RD, Wang Z, Cavasotto CN, Hittelman AB, Rogatsky I, Logan SK \& Garabedian MJ 2008 Glucocorticoid receptor phosphorylation differentially affects target gene expression. Molecular Endocrinology 22 1754-1766.

Chrousos GP \& Kino T 2007 Glucocorticoid action networks and complex psychiatric and/or somatic disorders. Stress 10 213-219.

Cruz JC \& Tsai LH 2004 A Jekyll and Hyde kinase: roles for Cdk5 in brain development and disease. Current Opinion in Neurobiology 14 390-394.

Davies L, Karthikeyan N, Lynch JT, Sial EA, Gkourtsa A, Demonacos C \& Krstic-Demonacos M 2008 Cross talk of signaling pathways in the regulation of the glucocorticoid receptor function. Molecular Endocrinology 22 1331-1344.

Diorio D, Viau V \& Meaney MJ 1993 The role of the medial prefrontal cortex (cingulate gyrus) in the regulation of hypothalamic-pituitary-adrenal responses to stress. Journal of Neuroscience 13 3839-3847.

Flood DG, Finn JP, Walton KM, Dionne CA, Contreras PC, Miller MS \& Bhat RV 1998 Immunolocalization of the mitogen-activated protein kinases p42MAPK and JNK1, and their regulatory kinases MEK1 and MEK4, in adult rat central nervous system. Journal of Comparative Neurology $398373-392$.

Garcia A, Marti O, Valles A, Dal-Zotto S \& Armario A 2000 Recovery of the hypothalamic-pituitary-adrenal response to stress. Effect of stress intensity, stress duration and previous stress exposure. Neuroendocrinology 72 114-125.

Givalois L, Arancibia S \& Tapia-Arancibia L 2000 Concomitant changes in $\mathrm{CRH}$ mRNA levels in rat hippocampus and hypothalamus following immobilization stress. Molecular Brain Research 75 166-171.

Goujon E, Laye S, Parnet P \& Dantzer R 1997 Regulation of cytokine gene expression in the central nervous system by glucocorticoids: mechanisms and functional consequences. Psychoneuroendocrinology 22 S75-S80.

Grewal SS, York RD \& Stork PJ 1999 Extracellular-signal-regulated kinase signalling in neurons. Current Opinion in Neurobiology 9 544-553.

Hatami H, Oryan S, Semnanian S, Kazemi B, Bandepour M \& Ahmadiani A 2007 Alterations of BDNF and NT-3 genes expression in the nucleus paragigantocellularis during morphine dependency and withdrawal. Neuropeptides 41 321-328.

Herman JP \& Spencer R 1998 Regulation of hippocampal glucocorticoid receptor gene transcription and protein expression in vivo. Journal of Neuroscience 18 7462-7473.

Ismaili N \& Garabedian MJ 2004 Modulation of glucocorticoid receptor function via phosphorylation. Annals of the New York Academy of Sciences 1024 86-101.

Kino T, Ichijo T, Amin ND, Kesavapany S, Wang Y, Kim N, Rao S, Player A, Zheng YL, Garabedian MJ et al. 2007 Cyclin-dependent kinase 5 differentially regulates the transcriptional activity of the glucocorticoid receptor through phosphorylation: clinical implications for the nervous system response to glucocorticoids and stress. Molecular Endocrinology 21 $1552-1568$.
De Kloet ER \& Reul JM 1987 Feedback action and tonic influence of corticosteroids on brain function: a concept arising from the heterogeneity of brain receptor systems. Psychoneuroendocrinology 12 83-105.

Krstic MD, Rogatsky I, Yamamoto KR \& Garabedian MJ 1997 Mitogenactivated and cyclin-dependent protein kinases selectively and differentially modulate transcriptional enhancement by the glucocorticoid receptor. Molecular and Cellular Biology 17 3947-3954.

Laemmli UK 1970 Cleavage of structural proteins during the assembly of the head of bacteriophage T4. Nature 227 680-685.

Lowry OH, Rosebrough NJ, Farr AL \& Randall RJ 1951 Protein measurement with the Folin phenol reagent. Journal of Biological Chemistry $193265-267$.

Lu NZ \& Cidlowski JA 2006 Glucocorticoid receptor isoforms generate transcription specificity. Trends in Cell Biology 16 301-307.

Magarinos AM, Somoza G \& De Nicola AF 1987 Glucocorticoid negative feedback and glucocorticoid receptors after hippocampectomy in rats. Hormone and Metabolic Research 19 105-109.

Malkesman O, Maayan R, Weizman A \& Weller A 2006 Aggressive behavior and HPA axis hormones after social isolation in adult rats of two different genetic animal models for depression. Behavioural Brain Research 175 408-414.

Marlier LNJL, Patacchioli FR, Przio O, Bottone A, Di Grezia R, Borboni P, Lauro R \& Angelucci L 1996 Differential regulation of adrenal corticoid receptors in the hippocampus and spinal cord of adrenalectomized rats. Journal of Neuroscience Research 43 526-534.

Marmigere F, Givalois L, Rage F, Arancibia S \& Tapia-Arancibia L 2003 Rapid induction of BDNF expression in hippocampus during immobilization stress challenge in adult rats. Hippocampus 13 646-655.

Meller E, Shen C, Nikolao TA, Jensen C, Tsimberg Y, Chen J \& Gruen RJ 2003 Region-specific effects of acute and repeated restraint stress on the phosphorylation of mitogen-activated protein kinases. Brain Research $\mathbf{2 5}$ $57-64$.

Merino JJ, Cordero MI \& Sandi C 2000 Regulation of hippocampal cell adhesion molecules NCAM and L1 by contextual fear conditioning is dependent upon time and stressor intensity. European Journal of Neuroscience 12 3283-3290.

Mizoguchi K, Ishige A, Aburada M \& Tabira T 2003 Chronic stress attenuates glucocorticoid negative feedback: involvement of the prefrontal cortex and hippocampus. Neuroscience 119 887-897.

Morsink MC, Steenbergen PJ, Vos JB, Karst H, Joels M, De Kloet ER \& Datson NA 2006 Acute activation of hippocampal glucocorticoid receptors results in different waves of gene expression throughout time. Journal of Neuroendocrinology 18 239-252.

Nishi M \& Kawata M 2006 Brain corticosteroid receptor dynamics and trafficking: implications from live cell imaging. Neuroscientist $\mathbf{1 2}$ 119-133.

Orti E, Bodwell JE \& Munck A 1992 Phosphorylation of steroid hormone receptors. Endocrine Reviews 13 105-128.

Ortiz J, Harris HW, Guitart X, Terwilliger RZ, Haycock JW \& Nestler EJ 1995 Extracellular signal-regulated protein kinases (ERKs) and ERK kinase (MEK) in brain: regional distribution and regulation by chronic morphine. Journal of Neuroscience 15 1285-1297.

Rogatsky I, Waase CL \& Garabedian MJ 1998 Phosphorylation and inhibition of rat glucocorticoid receptor transcriptional activation by glycogen synthase kinase-3 (GSK-3). Species-specific differences between human and rat glucocorticoid receptor signaling as revealed through GSK-3 phosphorylation. Journal of Biological Chemistry 273 14315-14321.

Sanchez ER, Hirst M, Scherrer LC, Tang HY, Welsh MJ, Harmon JM, Simons SS, Ringold GM \& Pratt WB 1990 Hormone-free mouse glucocorticoid receptors overexpressed in Chinese hamster ovary cells are localized to the nucleus and are associated with both hsp70 and hsp90. Journal of Biological Chemistry 265 20123-20130.

Sanchez MM, Aguado F, Sanchez-Toscano F \& Saphier D 1998 Neuroendocrine and immunocytochemical demonstrations of decreased hypothalamo-pituitary-adrenal axis responsiveness to restraint stress after long-term social isolation. Endocrinology 139 579-587. 
Sapolsky RM, Romero LM \& Munck A 2000 How do glucocorticoids influence stress responses? Integrating permissive, suppressive, stimulatory and preparative action Endocrine Reviews 21 55-89.

Schulkin J, Gold PW \& McEwen BS 1998 Induction of corticotropin releasing hormone gene expression by glucocorticoids: implication for understanding the states of fear and anxiety and allostatic load. Psychoneuroendocrinology 23 219-243.

Schulte-Herbruggen O, Chourbaji S, Ridder S, Brandwein C, Gass P, Hortnagl H \& Hellweg R 2006 Stress-resistant mice overexpressing glucocorticoid receptors display enhanced BDNF in the amygdala and hippocampus with unchanged NGF and serotonergic function. Psychoneuroendocrinology 31 1266-1277.

Shen CP, Tsimberg Y, Salvadore C \& Meller E 2004 Activation of Erk and JNK MAPK pathways by acute swim stress in rat brain regions. $B M C$ Neurosience 20 1-13.

Spencer RL, Kalman BA, Cotter CS \& Deak T 2000 Discrimination between changes in glucocorticoid receptor expression and activation in rat brain using western blot analysis. Brain Research 868 275-286.

Sweatt JD 2001 The neuronal MAP kinase cascade: a biochemical signal integration system subserving synaptic plasticity and memory. Journal of Neurochemistry 76 1-10.

Tapia-Arancibia L, Rage F, Givalois L \& Arancibia S 2004 Physiology of BDNF: focus on hypothalamic function. Frontiers in Neuroendocrinology 25 77-107.
Tsai LH, Delalle I, Caviness VS Jr, Chae T \& Harlow E 1994 p35 is a neuralspecific regulatory subunit of cyclin-dependent kinase 5. Nature $\mathbf{3 7 1}$ 419-423.

Webster JC, Jewell CM, Bodwell JE, Munck A, Sar M \& Cidlowski JA 1997 Mouse glucocorticoid receptor phosphorylation status influences multiple functions of the receptor protein. Journal of Biological Chemistry 272 9287-9293.

Weigel NL \& Moore NL 2007 Steroid receptor phosphorylation: a key modulator of multiple receptor functions. Molecular Endocrinology 21 2311-2319.

Wren AM, Small CJ, Abbott CR, Jethwa PH, Kennedy AR, Murphy KG, Stanley SA, Zollner AN, Ghatei MA \& Bloom SR 2002 Hypothalamic actions of neuromedin U. Endocrinology 143 4227-4234.

Zeng N, Athmann C, Kang T, Walsh JH \& Sachs G 1999 Role of neuropeptide-sensitive L-type $\mathrm{Ca}^{2+}$ channels in histamine release in gastric enterochromaffin-like cells. American Journal of Physiology. Gastrointestinal and Liver Physiology 277 1268-1280.

Received in final form 2 April 2009

Accepted 30 April 2009

Made available online as an Accepted Preprint 30 April 2009 